\title{
The mechanical strength of a ceramic porous hollow fiber
}

\author{
Patrick de Wit ${ }^{\mathrm{a}}$, Frederique S. van Daalen ${ }^{\mathrm{a}}$, Nieck E. Benes ${ }^{\mathrm{a}, *}$ \\ ${ }^{a}$ Films in Fluids, Department of Science and Technology, MESA+ Institute for Nanotechnology, University of Twente, P.O. Box 217, 7500 AE \\ Enschede, The Netherlands
}

\begin{abstract}
The mechanical strength of inorganic porous hollow fibers is a critical constraint that limits their wide scale application. Various methods, including 3-point bending, 4-point bending, and diametrical compression are used for the quantification of the mechanical strength. Here, we show that these methods cannot be used in an interchangeable manner. For large sets of alumina hollow fibers, the parameters describing the cumulative probability of failure functions depend on the type of measurement, i.e., 3 or 4-point, the span size, and the measurement geometry. This implies that reporting data on mechanical properties of inorganic hollow fibers requires that extensive information about the experimental details is provided, and that a direct quantitative comparison between datasets is unjustifiable. The mechanical strength of the alumina hollow fibers tends to follow a normal distribution, or log-normal distribution, instead of the often used Weibull distribution. Monte Carlo simulations demonstrate that, especially at small sample set sizes, it is difficult to accurately determine the shape of the probability distribution. However, detailed knowledge of the type and the shape of this distribution function is essential when mechanical strength values are to be used in further design.
\end{abstract}

Keywords: hollow fibers, failure analysis, mechanical strength, sample set size, statistical analysis

\section{Introduction}

Thin inorganic hollow fibers have large potential to be used as, for example, microfiltration membranes, catalyst supports, and (membrane) microreactors [1]. However, the widespread and large scale application of inorganic fibers is hampered by, in particular, their mechanical properties. Detailed knowledge of these properties is required for an acceptable comparison between different fibers, and quantification of the failure behaviour of fibers is of key importance for the design and construction of large area multi-fiber systems.

In ceramic reliability engineering, one often assumes a specific probability distribution. Based on the microstructure of a ceramic -being porous or non-porous- different probability distributions are assumed. In traditional nonporous ceramics the Weibull distribution is mostly used, whereas recently the use of the log-normal or normal distributions are proposed for porous ceramics [20].

\footnotetext{
${ }^{*}$ Corresponding author

Email address: N.E.Benes@utwente.nl (Nieck E. Benes)
} 
In addition to this, many methods are used to assess the mechanical robustness of inorganic fibers; most commonly their flexural or bending strength is determined via a 3-point [2-6] or 4-point bending test [7-10]. Alternatives include burst pressure measurements [11, 12] or diametrical compression tests [13, 14].

The reported mechanical strength is a direct result of the measurement method and the conditions used. As a result, comparison of strength data presented in literature can be deceptive. In addition to the measurement method, the amount of samples measured and the subsequent statistical analysis are of great importance.

The comparison of a 3-point versus a 4-point bending test is described in literature for various applications, like advanced dense ceramics [15-18] and polymers [19], but not for porous inorganic hollow fibers. In this paper, we demonstrate the pronounced influence of the measurement method on the reported strength value, and why it is crucial to not only report the measurement method, but also sample geometry and sample set size to allow comparison of reported strength values.

\section{Theoretical background}

\subsection{Strength distributions}

The result of fracture testing is usually reported as an average strength or mean strength of the measured stress at failure, of a set of $N$ samples.

$$
\sigma_{i}=\frac{\sum i=1 N \sigma_{i}}{N}
$$

A drawback of the average strength is that it contains no information about the spread of the strength values measured. In ceramics, defects are randomly distributed over the sample and they will vary in position, size, and severity. As a consequence, the strength of a fiber will vary from fiber to fiber, even for apparent identical fibers. As a measure for this spread often the standard deviation of the mechanical strength data is reported. In addition, if a small amount of samples is measured, the average strength might not be a good representation of the real strength number (this effect becomes more severe at lower sample sizes, e.g. $\mathrm{N}<10$ [21]). Therefore, the common interpretation of fracture tests is based on a statistical models that predict a probability of failure. It is often stated the one is required to measure at least 10-30 samples if the statistical model is known [18, 22], others propose that a minimum of 150-200 samples is required for an unknown model [21, 23].

The distribution parameters are of utmost importance in the design of components that consist of ceramics. In design, an acceptable probability of failure is selected, and the associated design stress is calculated using the statistical distribution function. Especially the lower tail of the probability distribution strongly affects the design stress [24]. Without appropriate characterization and the use of the correct statistical distribution, the measured strengths cannot be applied in design and might lead to erroneous conclusions. [20, 25-27]. 


\subsubsection{Weibull distribution}

The Weibull distribution [28] is the generally applied distribution for strength characterization of brittle ceramics with little defects. It is based on the so-called weakest link principle. In the majority of dense ceramics, only few defects are present. If the ceramic fails, it is assumed to fail at its weakest defect. The regular formulation of the Weibull distribution, used in measuring the strength of ceramics, is written as:

$$
P(\sigma)=1-\exp \left[-\left(\frac{\sigma}{\sigma_{\theta}}\right)^{m}\right]
$$

where $P(\sigma)$ is the cumulative probability of failure, $\sigma$ the applied stress, $m$ the Weibull modulus a measure for the spread of strength data and $\sigma_{\theta}$ is the characteristic strength. The characteristic strength and estimate of the Weibull modulus are often obtained via maximum likelihood fitting of the measured strength data. The Weibull characteristic strength depends on the test geometry, such type and size; and it is a value specific to a certain test.

An alternative representation of the probability of failure is the more general equation,

$$
P(\sigma, V)=1-\exp \left[-\int_{V}\left(\frac{\sigma}{\sigma_{0}}\right)^{m} d V\right]
$$

where $P(\sigma, V)$ is the cumulative probability of failure, $V$ is the volume of the component, $\sigma$ is the applied stress, $m$ is the Weibull modulus and $\sigma_{0}$ is the Weibull material scale parameter. If the integration in the above-mentioned equation is carried out the equation reduces to:

$$
P(\sigma)=1-\exp \left[-k V\left(\frac{\sigma}{\sigma_{0}}\right)^{m}\right]
$$

Where $k$ is a dimensionless constant that accounts for test specimen geometry and stress gradients. In general, $k$ is also a function of the estimated Weibull modulus. The product $k V$ is often referred to as the effective volume. Using this effective volume, the characteristic Weibull strength $\sigma_{\theta}$ can be converted into the Weibull material scale parameter using a relation such as

$$
\left(\sigma_{0}\right)_{V}=(k V)^{1 /(m)_{V}}\left(\sigma_{\theta}\right)_{V}
$$

This approach is discussed in detail in various references [22, 29, 30]. Calculation of the effective volume of a porous ceramic hollow fiber with its defects, pores, and macrovoids is problematic as the volume under stress is nearly impossible to estimate.

Mechanical strength testing combined with Weibull analysis are broadly standardized for dense ceramics, for example in ASTM C1161 [22] or DIN 843-5 [31]. Most methods recommend to measure at least 30 samples in order to accurately estimate the Weibull modulus and characteristic strength. The Weibull distribution, depending on the weakest link theory with non-interacting defects, is questioned to be suitable for certain ceramic strength data [26, 3235]. For example Danzer et al. [26] demonstrate that in certain situations a deviation of the Weibull distribution is expected; when the material exhibits a multi-model flaw size distribution (e.g., porosity), when defects interact, when $\mathrm{R}$-curve behavior is observed or when subcritical crack growth is likely [36]. Inorganic porous hollow fibers prepared 
by non-solvent induced phase inversion (NIPS) have a high defect density with a large range of defects such as pores, large finger like voids and agglomerates. This results in a questionable applicability of the Weibull model and its underlying assumption. Therefore, other models are also evaluated.

\subsubsection{Normal distribution}

The normal distribution is one of the mostly used distributions in sciences for real-valued random variables whose distributions are not known. Therefore, a normal distribution is used to describe the strength of brittle ceramics, in particular when these materials show a roughly symmetrical distribution and when the amount of defects is large $[33,37,38]$. Its parameters $\bar{\sigma}$ and $\alpha$ represent the mean and standard deviation of the distribution. The cumulative probability is given by:

$$
P(\sigma)=\frac{1}{2}\left[1+\operatorname{erf}\left(\frac{\sigma-\bar{\sigma}}{\alpha \sqrt{2}}\right)\right]
$$

\subsubsection{Log-normal distribution}

$\mathrm{Lu}$ et al. proposed the log-normal distribution for porous ceramics with high porosity. This assumes that the probability of a flaw being critical depends on a lot of factors such as size, shape and pore-grain interaction [20, 21]. The failure probability can be estimated by $p=\prod p_{i}$, where $p_{i}$ is the failure probability of the $i$-th factor of influence. Via $\ln p=\sum \ln p_{i}$ this results in an overall probability that follows the log-normal distribution [39]. The cumulative probability of a log-normal distribution is:

$$
P(\sigma)=\frac{1}{2}\left[1+\operatorname{erf}\left(\frac{\sigma-\bar{\sigma}}{\alpha \sqrt{2}}\right)\right]
$$

If data is log-normally distributed with parameters $\bar{\sigma}$ and $\alpha$, the logarithm of the data is distributed with the mean $\bar{\sigma}^{*}=\exp \left(\bar{\sigma}+\alpha^{2} / 2\right)$ and multiplicative standard deviation $s^{*}=\exp \left(2 \bar{\sigma}+\alpha^{2}\right)\left[\exp \left(\alpha^{2}\right)-1\right]$. Analog to the additive transformation for the normal distribution $\bar{\sigma} \pm \alpha$, the multiplicative transformation of the lognormal distribution can be expressed as $\bar{\sigma}^{*} \cdot / s^{*}$, where "./" indicates "times or divided" by [39].

\subsection{Minimum Information Criterion}

The unknown parameters of the proposed distribution functions are obtained by maximum likelihood method. Equation 8 shows the likelihood of a probability density function, where $\sigma_{i}$ is the strength of the $i$-th sample, $N$ is the total number of samples, $\mathcal{L}$ is the likelihood, and $f\left(\sigma_{i}\right)$ is the probability density function (pdf) of the proposed distribution [33].

$$
\ln (\mathcal{L})=\sum_{i=1}^{N} \ln f\left(\sigma_{i}\right)
$$

To compare the proposed models, the Akaike information criterion (AIC) [40] is used; which is an estimate for the distance between the true and the estimated distribution, and is defined as:

$$
A I C=-2 \ln (\mathcal{L})+2 k
$$


In which $\ln (\mathcal{L})$ is the maximum log-likelihood for a given model and $k$ is the number of parameters to be fitted. The $A I C$ values allow direct comparison, and a lower value for a certain distribution would indicate that this distribution is more likely, a difference in AIC value larger than 2 is considered statistically significant [21, 41].

\subsection{Anderson-Darling Goodness of Fit test}

In addition to the Akaike Information Criterion, an Anderson-Darling goodness of fit test is conducted. The Anderson-Darling test (AD), is one of the most powerful goodness of fit tests [42, 43] with critical values for various distributions being available in literature $[44,45]$. The hypothesis regarding the distributional form is rejected at the chosen confidence level $(\alpha=0.05)$ if the test statistic is greater than the critical value.

\subsection{Measurement methods}

Various methods and geometries are used to assess the flexural strength of ceramic hollow fiber membranes, all with their own distinct features. In current literature the 3-point and 4-point bending test are used interchangeably, both with various inner and outer span sizes [8, 46-48]. Recently the use of diametric compression tests is reported to assess the strength of inorganic hollow fibers [13, 49]. The reported strength value depends strongly on the measurement method and span size $[18,22,50]$.

\subsubsection{4-point bending test}

In the 4-point bending test the maximum stress is constant over a relatively large area between the two inner rollers, separated by distance $L_{i n}$. The maximum bending strength is computed using Equation 10 , where $F_{j}$ is the force at fracture for specimen $j, L_{\text {outer }}$ the distance between the two outer rollers, $K$ being half the distance between the inner and outer roller $\left(K=0.5\left(L_{\text {out }}-L_{\text {in }}\right)\right)$ and $d_{\text {out }, j}$ and $d_{i n, j}$ the outer and inner diameter of specimen $j$ :

$$
\sigma_{f, j}=\frac{16 F_{j} K d_{o u t, j}}{\pi\left(d_{o u t, j}^{4}-d_{i n, j}^{4}\right)}
$$

\subsubsection{3-point bending test}

The 3-point bending test exposes only a very small portion of the fiber to the maximum stress [16]. Therefore, bending strengths obtained via 3-point bending tests are likely to be larger as compared to ones obtained by means of the 4-point bending tests [19]. The maximum bending strength is computed using Equation 11 , where $F_{j}$ is the force at fracture for specimen $j, L_{\text {out }}$ the distance between the two outer rollers and $d_{o u t, j}$ and $d_{i n, j}$ the outer and inner diameter of specimen $j$, respectively.

$$
\sigma_{f, j}=\frac{8 F_{j} L_{o u t} d_{o u t, j}}{\pi\left(d_{o u t, j}^{4}-d_{i n, j}^{4}\right)}
$$

The major difference between the 3 and 4-point bending test is the location of the maximum bending moment and as a result the maximum stress. In a 4-point bending test a uniform stress profile is observed between the two inner rollers, whilst during a 3-point bending test the stress is concentrated under the loading roller. This influences the area under 
stress, which might result in a higher chance of observing a defect in the sample. The 3-point bending test has some advantages when compared to the 4-point bending test; it uses a simpler test fixture-minimizing the chance of roller misalignment- and a lower force to break the sample. Nonetheless, the ASTM recommends the 4-point bending test for most characterization purposes, mainly because a larger area is subjected to the maximum stress [18].

\subsubsection{Diametrical compression test}

In a diametrical compression test a compressive load is applied by two plates parallel to the axis of the fiber. The expression for the stress distribution is derived from the curved beam theory; if a fiber is subjected to a load $F_{j}$ over a length $L_{\text {out }}$, the resultant hoop stress is a sum of the stress components caused by axial and bending load. According to de With [51], the strength can be computed using Equation 12 where $F_{j}$ is the force at fracture, $r_{m}$ is the mean radius $\left(d_{o u t, j}+d_{i n, j}\right) / 4$, and $t$ is the wall thickness $\left(d_{o u t, j}-d_{i n, j}\right) / 2$.

$$
\sigma_{f, j}=\frac{2 F_{j} r_{m}\left(6 r_{m}-t\right)}{\pi L_{\text {out }} t^{2}\left(2 r_{m}-t\right)}
$$

Equation 12 assumes the fiber is a concentric ring structure and does not account for any eccentricity of the fiber. Although this measurement method is fairly simple to operate, the resultant strength values depend largely on the fibers geometry. De With does not recommend the use of this test, but proposed the use of a modified burst pressure [51-53]. The use of a burst pressure method is considered to be beyond the scope of this paper. For comparison reasons the diametrical compression test is included in this study, as it is recently used in the field of porous inorganic hollow fibers [13].

\section{Experimental}

\subsection{Hollow fiber fabrication}

Alumina hollow fibers were prepared by non-solvent induced phase separation (NIPS) using a mixture of AKP30 $\alpha$ - $\mathrm{Al}_{2} \mathrm{O}_{3}$ powder $\left(\mathrm{Al}_{2} \mathrm{O}_{3}\right.$, particle size of $0.3 \mu \mathrm{m}$, Sumitomo Chemicals Co. LTD. Japan), Polyethersulfone (PES, Ultrason, 6020P, BASF, Germany) and N-methylpyrrolidone (NMP, 99.5 wt\%, Sigma Aldrich, The Netherlands). Polyvinylpyrrolidone $\left(M_{w}\right.$ 1,300,000, Sigma Aldrich, The Netherlands) was used as viscosity enhancer and deionized water ( $>18 \mathrm{M} \Omega \mathrm{cm}^{-1}$ Milli-Q Advantage A10, Millipore) was used as non-solvent. Prior to use, PES and AKP-30 were dried overnight at $80^{\circ} \mathrm{C}$; all other chemicals were used as received. Table 1 describes the spinning conditions and recipe and fibers were prepared using standard methods [7].

After drying, the fibers were thermally treated under air $\left(100 \mathrm{~mL} \mathrm{~min}^{-1}\right)$ using a tubular furnace (STF16/610, Carbolite). The thermal treatment program consisted of $60 \mathrm{~min}$ at $300^{\circ} \mathrm{C}$ and $120 \mathrm{~min}$ at $1400{ }^{\circ} \mathrm{C}$. A heating and cooling rate of $5^{\circ} \mathrm{C} \min ^{-1}$ was used. After thermal treatment, the fibers were cut to the desired length. 

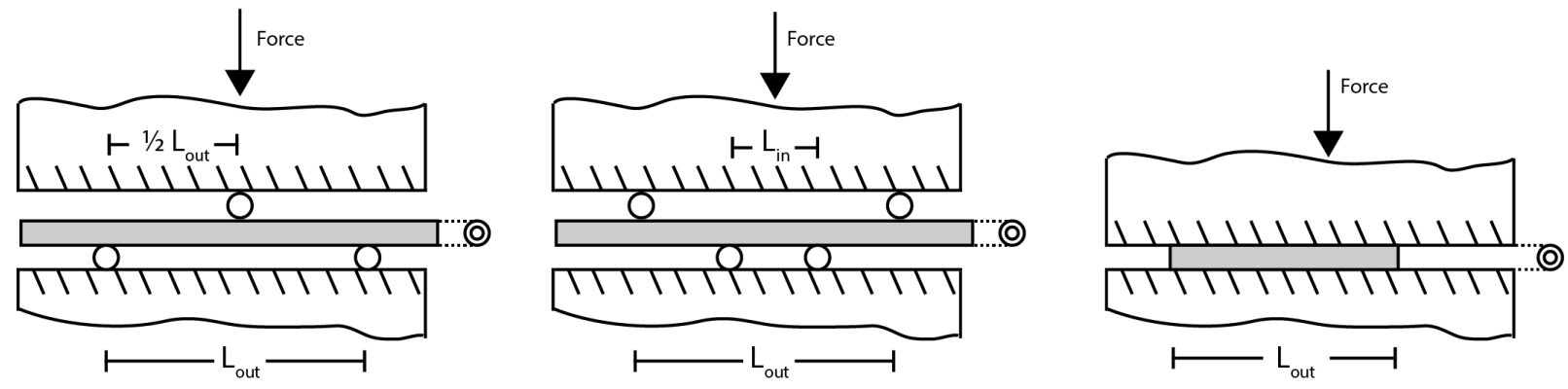

Figure 1: Schematic representation of the three different testing geometries. A: 3-point bending setup, B: 4-point bending $\operatorname{setup}\left(L_{\text {in }}=0.5 L_{\text {out }}\right)$, C: Diametrical compression setup

\subsection{Experimental setup}

The bending strength of the alumina hollow fibers was measured at room temperature $\left(20 \pm 2{ }^{\circ} \mathrm{C}\right)$ and a relative humidity of $40 \% \pm 10 \%$, using a 5564A mechanical testing bench (Instron) equipped with a $100 \mathrm{~N}$ load cell. All testing was carried out according to ASTM C1684-08 [18], which has been adapted at the following points:

- Rollers are not free to move laterally

- A tube is used instead of a rod

- The ceramic material is porous

Figure 1 schematically displays the three different setups used. In order to make a fair comparison $L_{\text {out }}$ was kept constant for both the 3-point and the 4-point bending tests. For diametrical compression testing, the fibers length was measured prior to each experiment.

A piece of hollow fiber was loaded into the test fixture. The load was measured at constant extension rate $\left(2 \mathrm{~mm} \mathrm{~min}^{-1}\right)$. The inner and outer diameter of the fiber were measured, after fracture, using a digital caliper (Mitutoyo CD-15CPX, $\Delta d \pm 0.02 \mathrm{~mm}$ ). All fibers were tested as-fabricated and no surface treatment was carried out on the fibers. Fibers originated from one spinning batch, but from different sintering batches. Fibers were taken randomly from different sintering batches for the various experiments. A sample set size of $N=100$ was used for the $20 \mathrm{~mm}$ outer span size and diametrical compression measurements. For the $40 \mathrm{~mm}$ outer span size experiments, a sample set size of $N=50$ was used. All fiber fragments were collected for post-failure analysis; for various fibers the fracture surface was investigated using SEM. In order to verify the accuracy of inner and outer diameter, obtained by the digital caliper, the inner and outer diameter were also estimated from the scanning electron micrographs. The differences in diameters were less than 5 percent (see the Supplementary Information). 


\begin{tabular}{ll}
\hline Condition & Value \\
\hline PES & $9.40 \%$ \\
NMP & $40.0 \%$ \\
AKP-30 & $48.9 \%$ \\
PVP K-95 & $0.70 \%$ \\
Water & $1.00 \%$ \\
Bore Liquid & $\mathrm{H}_{2} \mathrm{O}$ \\
Coagulation bath & $\mathrm{H}_{2} \mathrm{O}$ \\
Extrusion pressure & $2 \mathrm{bar}$ \\
Air gap & $3 \mathrm{~cm}$ \\
Bore liquid flow rate & $7 \mathrm{~mL} \mathrm{~min}{ }^{-1}$ \\
Diameter spinneret & $\mathrm{OD} / \mathrm{ID}=2.0 \mathrm{~mm} / 0.8 \mathrm{~mm}$ \\
Drying after spinning & $>24 \mathrm{~h}$ \\
\hline
\end{tabular}

\section{Results and discussion}

Figure 2 shows a cross-sectional scanning electron micrograph of the $\mathrm{Al}_{2} \mathrm{O}_{3}$ fiber after sintering, where it is clear that the wall of the fiber exhibits an asymmetrical structure. Upon further magnification (Fig $2 \mathrm{~B}$ and $\mathrm{C}$ ) the macro-voids in the wall and the porous structure in between the sintered alumina grains can be seen. The fibers show an average pore diameter of $300 \mathrm{~nm}$ (Mercruy Intrusion), a porosity of approximately 50 percent (He and $\mathrm{Hg}$ density measurement) and a clean water permeability of approximately $500 \mathrm{~L} \mathrm{~m}^{-2} \mathrm{~h}^{-1} \mathrm{bar}^{-1}$ (see the Supplementary Information for further details about the fiber characterization.)
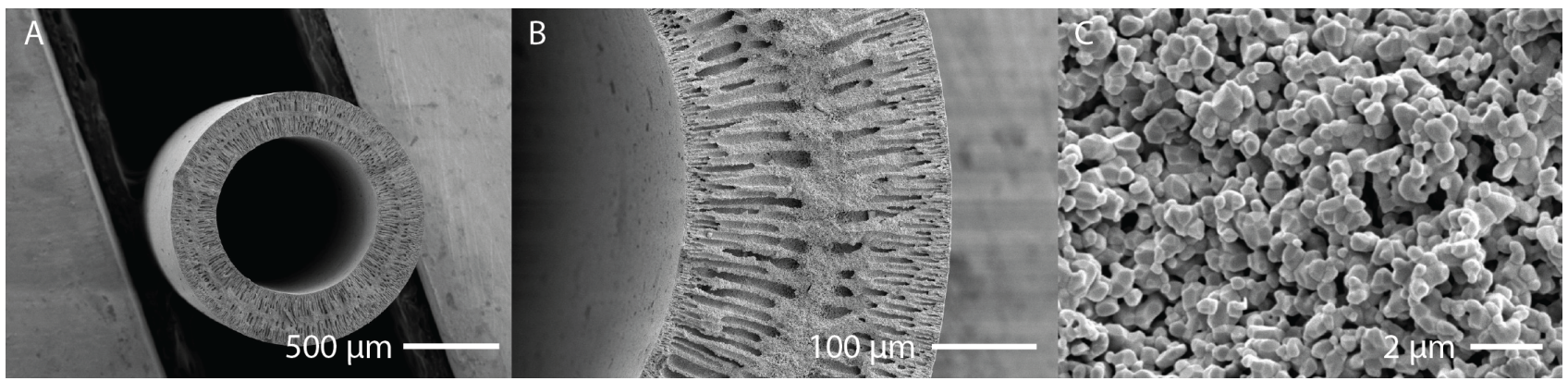

Figure 2: Cross-sectional scanning electron micrographs of a sintered aluminum oxide fiber used in mechanical testing. A: 50x magnification showing the roundness of the fiber B: close-up of fiber wall showing the asymmetrical wall structure with the presence of large macro-voids C: detail of individual grains in the fiber wall. 


\subsection{Mechanical strength}

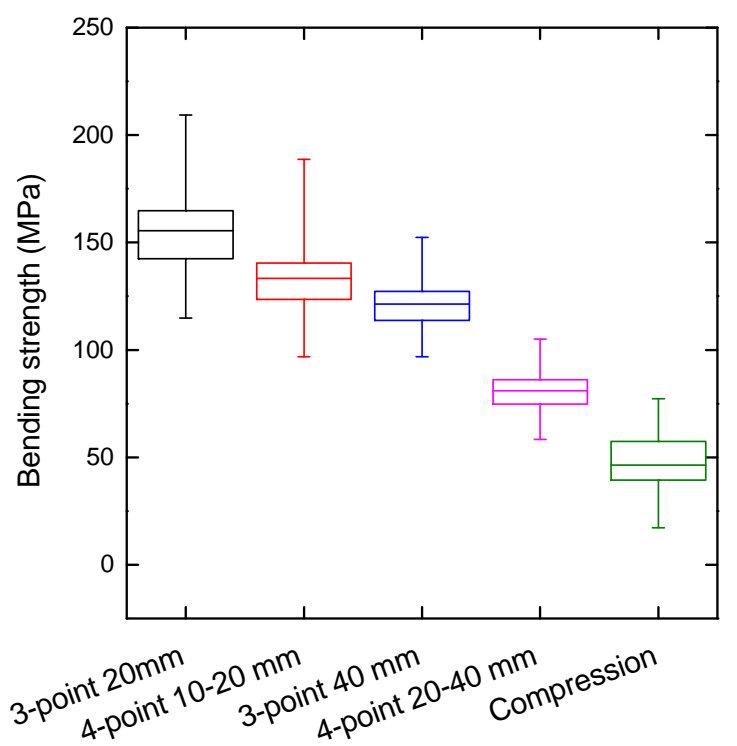

Figure 3: Summary of bending strengths measured using various methods. The lower and upper error bar denote the minimal and maximal observed mechanical strength, the boundaries of the box indicate the $I Q R$, whereas the horizontal line represents the median value.

Figure 3 shows the observed mechanical strengths obtained by the 3 different methods; the 3-point bending test, the 4-point bending test and the diametrical compression. A detailed overview of all results, including inner and outer diameter, location of fracture, and load/extension can be found in the Supplementary Datafile [54]. In Figure 3 the lower and upper error bar denote the lowest and highest observed strength. The box indicates the inter quartile range $(I Q R)$ of the observed mechanical strengths, with the horizontal line representing the median value. For all experiments a large spread in the measured strength is observed. Furthermore, the difference between the 3-point and 4-point bending test and the effect of the outer span size $L_{\text {out }}$ is worth mentioning.

For both outer spans, the 4-point bending test results in a significantly lower observed strength as compared to the 3-point bending test. This effect is well-described in literature [15, 18, 19], where it is indicated that results obtained by 4-point bending test will be lower as compared to 3-point bending test results. A larger span size (20 versus $40 \mathrm{~mm}$ ) results in a significantly lower mechanical strength, this effect is visible for both 4 and 3-point bending tests. A 3-point bending test with an outer span of $20 \mathrm{~mm}$ results in a median strength of $\sim 155 \mathrm{MPa}$, the same experiment with $L_{\text {out }}=$ $40 \mathrm{~mm}$ results in a median strength of approximately $120 \mathrm{MPa}$. This size effect is often described in literature, where an increased volume under stress (for example due to increased span size) will result in lower strength numbers [55]. The diametrical compression test shows the lowest mechanical strength values of all tests, however a comparison to the bending tests is complicated. The stress profile introduced in this method is rather different as compared to bending tests, and Figure 3 clearly shows that one should be extremely cautious when comparing results obtained by this method to the results of a bending test. Matlab ${ }^{\circledR}$ is used to rank the measured strengths and to fit the three 
distributions to the data using maximum-likelihood methods. Table 2 displays the fitted parameters for all tests bending and compression- obtained by fitting the measured strength to a Weibull, Normal or Log-normal distribution. Figure 4 visualizes the different probability models jointly with the measured and ranked data of the four different bending tests (empirical cumulative probability of failure estimated using the Kaplan-Meier method [56]).

Figure 4 shows that the strength distributions tend to follow a normal or log-normal distribution. The Weibull distribution appears to be a lesser fit, especially at the lower end of the distribution.
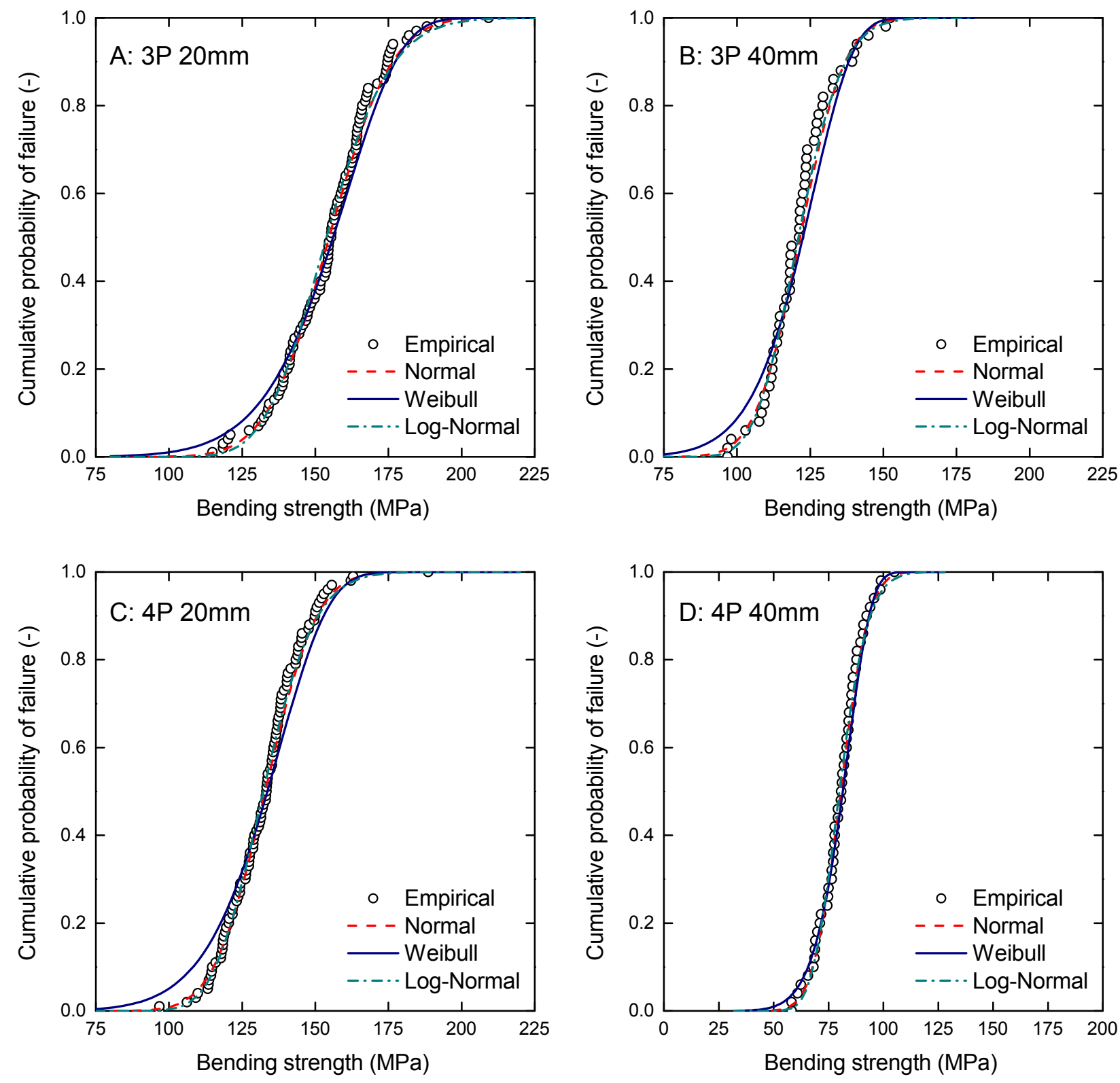

Figure 4: Cumulative probability of failure; empirical data (Kaplan-Meier estimate) and the 3 different distributions (parameters see Table 3). A: 3-point 20mm, B: 3-point 40mm, C: 4-point 10-20mm, D: 4-point 20-40mm 
As shown by the parameter estimates in Table 2, the difference between the mean value obtained from a normal distribution and the characteristic strength from a Weibull distribution can clearly be seen for all test geometries; the Weibull characteristic strength is always higher when compared to the mean value. The parameter that describes the shape of the probability distribution appears to independent of the span size of the test, with all shape parameters $(m$ for Weibull, $\alpha$ for normal and $s^{*}$ for lognormal) being the same order of magnitude for the same type of test.

However, the shape values for a 3-point bending test are in disagreement with the 4-point bending test, where the 4-point bending test suggests a more narrow distribution. This might be explained by the fact that for a 4-point bending test the area under stress is larger, hence a larger probability a specimen will fail, possibly resulting in a more narrow distribution. This is also seen in the values that represent the mean and characteristic strength; the larger the volume subject to stress; the lower the observed strength value. For example, a 3-point bending test with a span size of $20 \mathrm{~mm}$ displays a significantly larger strength number as compared to a 3-point bending test with a span size of $40 \mathrm{~mm}$, albeit the shape factor is not strongly influenced by the span size. Table 2 again demonstrates that values obtained using a diametrical compression test are an order of magnitude smaller as compared to bending tests. This originates from the different stress profile in the fiber.

Table 2: Fitted parameters for all 5 different tests using Weibull, Normal and Log-normal distributions. All parameters are shown with their 95\% confidence interval.

\begin{tabular}{llllll}
\hline Type & 3 point & 4 point & 3 point & 4 point & Compression \\
\hline Span size & $20 \mathrm{~mm}$ & $10-20 \mathrm{~mm}$ & $40 \mathrm{~mm}$ & $20-40 \mathrm{~mm}$ & N/A \\
$N$ & 100 & 100 & 50 & 50 & 100 \\
Weibull & & & & & \\
$\sigma_{\theta}$ & $165.3 \pm 3.5$ & $139.2 \pm 3.2$ & $127.1 \pm 3.7$ & $85.0 \pm 2.8$ & $53.3 \pm 2.6$ \\
$m$ & $9.45 \pm 1.2$ & $8.93 \pm 1.1$ & $9.98 \pm 1.8$ & $8.73 \pm 1.6$ & $4.22 \pm 0.6$ \\
Normal & & & & & \\
$\bar{\sigma}$ & $154.8 \pm 3.3$ & $132.9 \pm 2.7$ & $121.5 \pm 3.4$ & $80.6 \pm 2.8$ & $48.5 \pm 2.5$ \\
$\alpha$ & $16.7 \pm 2.0$ & $13.8 \pm 1.7$ & $12.0 \pm 1.9$ & $10.0 \pm 1.6$ & $12.6 \pm 1.5$ \\
Lognormal & & & & & \\
$\overline{\sigma^{*}}$ & $153.8 \pm 3.3$ & $132.2 \pm 2.7$ & $121.0 \pm 3.3$ & $80.0 \pm 2.8$ & $46.8 \pm 2.5$ \\
$s^{*}$ & $1.12 \pm 0.01$ & $1.11 \pm 0.01$ & $1.10 \pm 0.01$ & $1.13 \pm 0.02$ & $1.31 \pm 0.04$ \\
\hline
\end{tabular}

Table 3 shows the calculated $A I C$ values for all 3 distributions. For all bending tests the Weibull distribution is the least likely distribution $(\max A I C=$ Weibull). For example Danzer [26], or Lu [20] predicted this behavior for porous ceramics, as they state that a (log-) normal distribution can be expected. The results of the Anderson-Darling goodness of fit test are shown in Table 4, and indicate that the Weibull distribution appears to be the least likely distribution for the bending tests. 
Table 3: The difference in $A I C$ values calculated for the three distributions, $\triangle A I C$ is defined as $\max (A I C) \min (A I C)$

\begin{tabular}{lllll}
\hline Type & $N$ & $\min (A I C)$ & $\max (A I C)$ & $\triangle A I C$ \\
\hline 3-point $20 \mathrm{~mm}$ & 100 & Normal & Weibull & 11.23 \\
3-point $40 \mathrm{~mm}$ & 50 & LogNormal & Weibull & 11.12 \\
4 point 10-20 mm & 100 & LogNormal & Weibull & 26.00 \\
4 point 20-40mm & 50 & Normal & Weibull & 3.24 \\
Compression & 100 & Normal & LogNormal & 2.44 \\
\hline
\end{tabular}

Table 4: Anderson-Darling goodness-of-fit results for the various measurement methods.

\begin{tabular}{llllllllllll}
\hline & \multicolumn{3}{c}{ Normal } & \multicolumn{3}{c}{ Weibull } & & \multicolumn{3}{c}{ Lognormal } \\
& p-value & $\mathrm{TS}^{\mathrm{a}}$ & $\mathrm{CV}$ & $\mathrm{p}$-value & $\mathrm{TS}$ & $\mathrm{CV}$ & $\mathrm{p}$-value & $\mathrm{TS}$ & $\mathrm{CV}$ \\
\hline 3-point 20 mm & 0.5634 & 0.3140 & 0.7460 & 0.0112 & 1.0174 & 0.7538 & 0.1564 & 0.5484 & 0.7460 \\
3-point 40 mm & 0.5186 & 0.3308 & 0.7460 & $<0.0005$ & 2.1802 & 0.7538 & 0.6167 & 0.2948 & 0.7460 \\
4 point 10-20 mm & 0.1297 & 0.5765 & 0.7399 & 0.0007 & 1.4831 & 0.7478 & 0.4078 & 0.3743 & 0.7399 \\
4 point 20-40mm & 0.9758 & 0.1437 & 0.7399 & 0.4156 & 0.3781 & 0.7478 & 0.6944 & 0.2663 & 0.7399 \\
Compression & 0.1213 & 0.5925 & 0.7460 & 0.0524 & 0.7455 & 0.7538 & 0.5549 & 0.3171 & 0.7460 \\
\hline
\end{tabular}

${ }^{a}$ Test statistic, ${ }^{b}$ Critical Value

The differences in AIC value between the Normal and Lognormal distribution are usually below 2, making it impossible to determine whether the preferred distribution is Normal or Lognormal [40, 41], as in certain cases the Lognormal distribution is converges to the Normal distribution [39]. The same holds for the diametrical compression test, where the difference in $A I C$ values or the results of the Anderson-Darling test are too small to state that any distribution is more likely. In general, it is difficult to accurately assess the distribution of these measurements, no specific distribution stands out nor is rejected.

\subsection{Influence of sample set size}

The parameters of the experimental data, which are estimated using maximum likelihood method, are considered to be the "true" parameters representing the distribution. These values were used to generate virtual strength data using Monte Carlo (MC) methods [21, 57]. This was done using Matlab ${ }^{\circledR}$; the full details and the Matlab source code are given in the Supplementary Information. Based on the parameter estimates in Table 2, virtual strength data was generated for a 4-point bending test $\left(L_{\text {out }}=20 \mathrm{~mm}\right)$, assuming a normal strength distribution. Out of the virtual strength data; sample sets are taken with a variable sample set size $N$. This is done 10.000 times, resulting in 10.000 sample sets of size $N$. These sample sets are used to determine new distribution parameters (mean strength and standard deviation) that describe this specific sample set of size $N$ shows the effect of sample set size on the 

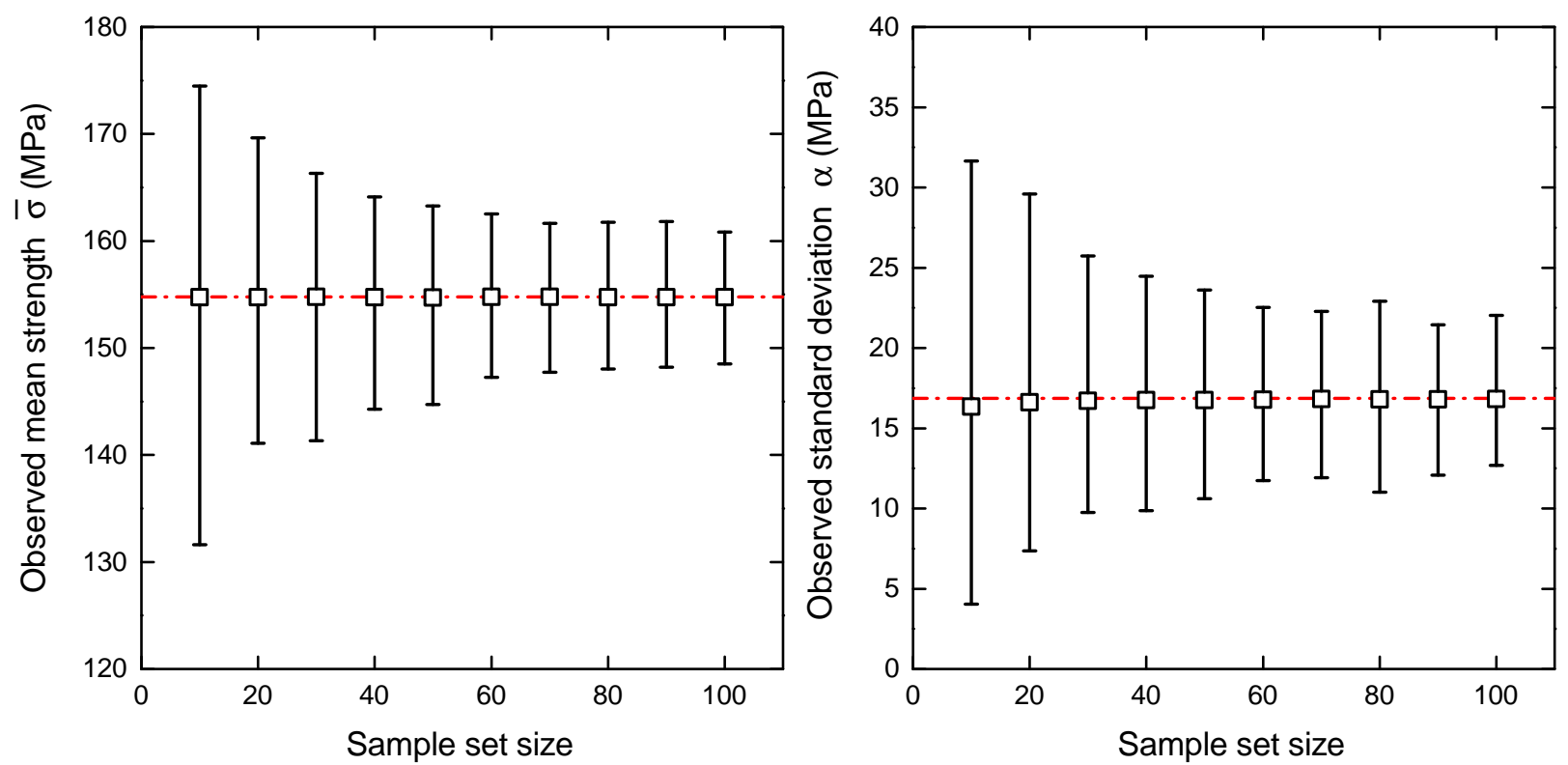

Figure 5: Effect of sample size on mean strength (a, left) and standard deviation (b, right) for 4-point bending, 20mm span, normal distribution. Error bars represent observed minimal and maximal values for mean (a) and standard deviation (b).

mean strength (a) and standard deviation (b). The error bars represent the range of values observed in 10,000 MC simulations.

The observed mean and standard deviation in the 10.000 virtual sample sets varies substantially, especially for the lower sample set sizes. The variation on the observed mean would result in an over- or underestimation of the average strength of a fiber, however the variation of the observed standard deviation would result in a different shape of the probability distribution. At larger sample set sizes, the observed mean and standard deviation converge to the "true" parameters representing the distribution. Nonetheless, even at fair sample set sizes of $N=100$ the observed values differ significantly from the true parameters. This shows that the amount of samples measured is solely depending on what is economically/practically viable [21]. If one would use the parameters obtained by fitting 10 test specimens, Figure 6 shows the cumulative probability of failure a sample for two extreme cases, one where the lowest estimate for the standard deviation is used, whereas the second one uses the highest standard deviation estimate. The minimal and maximal values for the standard deviation are taken from Figure 5. For the mean strength the "true" mean strength is used. The large difference in standard deviation -which is a measure for the spread of the distribution- results in tremendous over- or underestimation of both the higher and lower end of the probability of failure. 


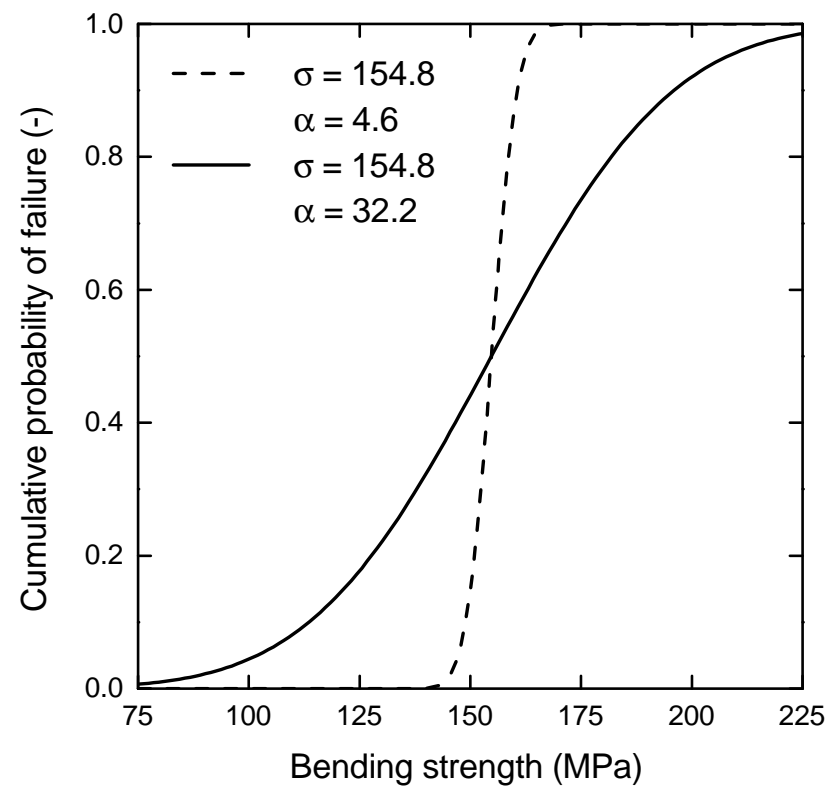

Figure 6: Cumulative probability of failure if the extreme values for the standard deviation are used to predict failure behaviour

In the design of ceramic compounds an acceptable probability of failure is selected prior to design, depending on the specific application and possible consequences of failure. If one assumes an acceptable failure probability of 0.01 this would mean that at the corresponding stress, 1 out of 100 fibers is likely to fail. From Figure 6 it shows that with an allowable probability of failure of 0.01 , the stress would be $80 \mathrm{MPa}$ or $148 \mathrm{MPa}$, depending on the used standard deviation[24]. If one would erroneous estimate the type of distribution and its associated parameters; it would lead to wrong assumptions in design and possibly premature failure of, for example, a membrane module. This demonstrates that, especially for design, it is of key importance to measure sufficient samples in order to accurately describe the shape of the distribution.

\section{Conclusion}

The mechanical strength of porous alumina hollow fiber membranes was measured using different measurement geometries. The resultant mechanical strength number is a direct result of the measurement method. Values obtained by 3-point bending tests will be higher as compared to values obtained by 4-point bending test. Mechanical strength numbers obtained by diametrical compression are nearly a factor 2 lower as compared to the bending test numbers. All bending methods are suitable to characterize the mechanical strength of a ceramic hollow fiber, however to allow a direct comparison it is highly recommended to stick to span sizes and geometries mentioned in various standards, such as in the ASTM C1684-08 [18]. The utilized measurement method, span size and sample set size should be reported, combined with the mean strength and standard deviation. Only when these values are reported a comparison between different types of fibers can be made. The minimum required sample set size depends largely on the purpose 
of the measurements, in order to characterize the mechanical strength and to make a comparison to other fibers, 20 to 30 samples will give reasonable estimates of the average mechanical strength and standard deviation. If the values are used for design, detailed knowledge of the underlying probability distribution is required. In order to determine this, 100 or even more samples should be measured. Only at these large sample set sizes it is possible to determine the distribution type. Detailed knowledge about the distribution type and distribution parameters is required as the specific shape of this distribution largely affects the design strength.

\section{Acknowledgements}

This research is supported by the Dutch Technology Foundation STW, which is part of the Netherlands Organisation for Scientific Research (NWO), and which is partly funded by the Ministry of Economic Affairs.

\section{References}

[1] I. Moch, Hollow-Fiber Membranes, in: Kirk-Othmer Encyclopedia of Chemical Technology, John Wiley \& Sons, Inc., 2000.

[2] X. Tan, N. Liu, B. Meng, S. Liu, Morphology control of the perovskite hollow fibre membranes for oxygen separation using different bore fluids, J. Membr. Sci. 378 (2011) 308-318.

[3] S. Liu, K. Li, R. Hughes, Preparation of porous aluminium oxide $\left(\mathrm{Al}_{2} \mathrm{O}_{3}\right)$ hollow fibre membranes by a combined phase-inversion and sintering method, Ceram. Int. 29 (2003) 875-881.

[4] D. Chen, M. Sixta, X. Zhang, L. De Jonghe, R. Ritchie, Role of the grain-boundary phase on the elevated-temperature strength, toughness, fatigue and creep resistance of silicon carbide sintered with Al, B and C, Acta Mater. 48 (2000) 4599-4608.

[5] Z. Shi, Y. Zhang, C. Cai, C. Zhang, X. Gu, Preparation and characterization of $\alpha-\mathrm{Al}_{2} \mathrm{O}_{3}$ hollow fiber membranes with four-channel configuration, Ceram. Int. 41 (2015) 1333-1339.

[6] Y.-L. Elaine Fung, H. Wang, Investigation of reinforcement of porous alumina by nickel aluminate spinel for its use as ceramic membrane, J. Membr. Sci. 444 (2013) 252-258.

[7] M. W. J. Luiten-Olieman, M. J. T. Raaijmakers, L. Winnubst, T. C. Bor, M. Wessling, A. Nijmeijer, N. E. Benes, Towards a generic method for inorganic porous hollow fibers preparation with shrinkage-controlled small radial dimensions, applied to $\mathrm{Al}_{2} \mathrm{O}_{3}, \mathrm{Ni}, \mathrm{SiC}$, stainless steel, and YSZ, J. Membr. Sci. 407-408 (2012) 155-163.

[8] P. de Wit, E. J. Kappert, T. Lohaus, M. Wessling, A. Nijmeijer, N. E. Benes, Highly permeable and mechanically robust silicon carbide hollow fiber membranes, J. Membr. Sci. 475 (2015) 480-487.

[9] V. Middelkoop, H. Chen, B. Michielsen, M. Jacobs, G. Syvertsen-Wiig, M. Mertens, A. Buekenhoudt, F. Snijkers, Development and characterisation of dense lanthanum-based perovskite oxygen-separation capillary membranes for high-temperature applications, J. Membr. Sci. 468 (2014) 250-258.

[10] C. Buysse, A. Kovalevsky, F. Snijkers, A. Buekenhoudt, S. Mullens, J. Luyten, J. Kretzschmar, S. Lenaerts, Development, performance and stability of sulfur-free, macrovoid-free BSCF capillaries for high temperature oxygen separation from air, J. Membr. Sci. 372 (2011) 239-248.

[11] J. Smid, C. G. Avci, V. Günay, R. A. Terpstra, J. P. G. M. Van Eijk, Preparation and characterization of microporous ceramic hollow fibre membranes, J. Membr. Sci. 112 (1996) 85-90.

[12] I. Voigt, G. Fischer, P. Puhlfürß, M. Schleifenheimer, M. Stahn, TiO 2 -NF-membranes on capillary supports, Sep. Purif. Technol. 32 (2003) 87-91.

[13] M. Lee, Z. Wu, R. Wang, K. Li, Micro-structured alumina hollow fibre membranes: Potential applications in wastewater treatment, J. Membr. Sci. 461 (2014) 39-48. 
[14] T. Byun, E. Lara-Curzio, R. Lowden, L. Snead, Y. Katoh, Miniaturized fracture stress tests for thin-walled tubular SiC specimens, J. Nucl. Mater. 367-370 (2007) 653-658.

[15] S. A. Rodrigues, J. L. Ferracane, A. Della Bona, Flexural strength and Weibull analysis of a microhybrid and a nanofill composite evaluated by 3- and 4-point bending tests., Dent. Mater. 24 (2008) 426-31.

[16] F. Mujika, On the difference between flexural moduli obtained by three-point and four-point bending tests, Polym. Test. 25 (2006) $214-220$.

[17] G. Quinn, R. Morrell, Design data for engineering ceramics: a review of the flexure test, J. Am. Ceram. Soc. 74 (1991).

[18] ASTM, C1684-08 Standard Test Method for Flexural Strength of Advanced Ceramics at Ambient Temperature, ASTM International, West Conshohocken, PA (2002) 1-21.

[19] P. Chitchumnong, S. Brooks, G. Stafford, Comparison of three-and four-point flexural strength testing of denture-base polymers, Dent. Mater. (1989) 3-6.

[20] C. Lu, A reassessment of the strength distributions of advanced ceramics, Journal of the Australian Ceramic Society 44 (2008) $38-41$.

[21] S. Nohut, Influence of sample size on strength distribution of advanced ceramics, Ceram. Int. 40 (2014) 4285-4295.

[22] ASTM, C1239-13 Standard Practice for Reporting Uniaxial Strength Data and Estimating Weibull Distribution Parameters for Advanced Ceramics, ASTM International, West Conshohocken, PA (2014) 1-18.

[23] T. Hoshide, H. Sugiyama, Numerical Analysis of Sample-Size Effect on Strength of Alumina, J. Mater. Eng. Perform. 22 (2012) 1-8.

[24] R. Danzer, On the relationship between ceramic strength and the requirements for mechanical design, J. Eur. Ceram. Soc. 34 (2014) $3435-3460$

[25] R. Danzer, A general strength distribution function for brittle materials, J. Eur. Ceram. Soc. 10 (1992) $461-472$.

[26] R. Danzer, P. Supancic, J. Pascual, T. Lube, Fracture statistics of ceramics: Weibull statistics and deviations from Weibull statistics, Engineering Fracture Mechanics 74 (2007) 2919-2932.

[27] C. A. Klein, Characteristic strength, Weibull modulus, and failure probability of fused silica glass, Opt. Eng. 48 (2009) 113401.

[28] W. Weibull, A statistical distribution function of wide applicability, Journal of applied Mechanics 18 (1951) $293-297$.

[29] G. D. Quinn, Weibull Effective Volumes and Surfaces for Cylindrical Rods Loaded in Flexure, Journal of American Ceramic Society 86 (2003) 475-479.

[30] Effective area and effective volume calculations for ceramic test specimens, volume 1 of Power for Land, Sea, and Air, ASME Turbo Expo 2009:, 2009.

[31] DIN, Advanced technical ceramics - Mechanical properties of monolithic ceramics at room temperature - Part 5: Statistical analysis, 2007.

[32] R. Danzer, Some notes on the correlation between fracture and defect statistics: Are Weibull statistics valid for very small specimens?, J. Eur. Ceram. Soc. 26 (2006) 3043-3049.

[33] C. Lu, R. Danzer, F. Fischer, Fracture statistics of brittle materials: Weibull or normal distribution, Physical Review E 65 (2002) 067102.

[34] F. Chen, R. Mourhatch, T. T. Tsotsis, M. Sahimi, Experimental studies and computer simulation of the preparation of nanoporous siliconcarbide membranes by chemical-vapor infiltration/chemical-vapor deposition techniques, Chem. Eng. Sci. 63 (2008) 1460-1470.

[35] B. Basu, D. Tiwari, D. Kundu, R. Prasad, Is Weibull distribution the most appropriate statistical strength distribution for brittle materials?, Ceram. Int. 35 (2009) 237-246.

[36] A. Zimmermann, J. Rödel, Fracture Statistics Based on Pore/Grain-Size Interaction, J. Am. Ceram. Soc. 82 (2004) $2279-2281$.

[37] R. H. Doremus, Fracture statistics: A comparison of the normal, Weibull, and Type I extreme value distributions, J. Appl. Phys. 54 (1983) 193.

[38] B. Stawarczyk, M. Ozcan, C. H. F. Hämmerle, M. Roos, The fracture load and failure types of veneered anterior zirconia crowns: an analysis of normal and Weibull distribution of complete and censored data., Dent. Mater. 28 (2012) 478-87.

[39] E. Limpert, W. a. Stahel, M. Abbt, Log-normal Distributions across the Sciences: Keys and Clues, Bioscience 51 (2001) 341.

[40] H. Akaike, A new look at the statistical model identification, Automatic Control, IEEE Transactions on (1974).

[41] J. Frank, Electron Tomography: Methods for Three-Dimensional Visualization of Structures in the Cell, volume 40, 2 nd edition, 2006.

[42] N. M. Razali, Y. B. Wah, Power comparisons of Shapiro-Wilk, Kolmogorov-Smirnov, Lilliefors and Anderson-Darling tests, Journal of 
Statistical Modeling and Analytics 2 (2011) 21-33.

[43] T. Anderson, D. Darling, Asymptotic theory of certain goodness of fit criteria based on stochastic processes, The annals of mathematical statistics 23 (1952) 193-212.

[44] M. Stephens, Asymptotic results for goodness-of-fit statistics with unknown parameters, The Annals of Statistics 4 (1976) $357-369$.

[45] J. G. Bush, B. W. Woodruff, A. H. Moore, E. J. Dunne, Modified cramer-von mises and anderson-darling tests for weibull distributions with unknown location and scale parameters, Communications in Statistics - Theory and Methods 12 (1983) $2465-2476$.

[46] M. Luiten-Olieman, L. Winnubst, A. Nijmeijer, M. Wessling, N. E. Benes, Porous stainless steel hollow fiber membranes via dry-wet spinning, J. Membr. Sci. 370 (2011) 124-130.

[47] S. Husain, W. J. Koros, Macrovoids in Hybrid Organic/Inorganic Hollow Fiber Membranes, Industrial \& Engineering Chemistry Research 48 (2009) 2372-2379.

[48] X. Zhang, D. K. Wang, D. R. S. Lopez, J. C. Diniz da Costa, Fabrication of nanostructured TiO 2 hollow fiber photocatalytic membrane and application for wastewater treatment, Chemical Engineering Journal 236 (2014) 314-322.

[49] Z. Wu, R. Faiz, T. Li, B. F. Kingsbury, K. Li, A controlled sintering process for more permeable ceramic hollow fibre membranes, J. Membr. Sci. 446 (2013) 286-293.

[50] D. J. Green, An introduction to the mechanical properties of ceramics, Cambridge University Press, 1998.

[51] G. de With, Note on the use of the diametral compression test for the strength measurement of ceramics, Journal of materials science letters 3 (1984) 6-8.

[52] R. Sedlacek, F. a. Halden, Method for Tensile Testing of Brittle Materials, Rev. Sci. Instrum. 33 (1962) 298.

[53] J. de Jong, N. Benes, G. Koops, M. Wessling, Towards single step production of multi-layer inorganic hollow fibers, J. Membr. Sci. 239 (2004) 265-269.

[54] P. de Wit, Raw data, 2015.

[55] S.-D. Pang, Z. P. Bažant, J.-L. Le, Statistics of strength of ceramics: finite weakest-link model and necessity of zero threshold, International Journal of Fracture 154 (2009) 131-145.

[56] E. L. Kaplan, P. Meier, Nonparametric Estimation from Incomplete Observations, J. Am. Stat. Assoc. 53 (1958) $457-481$.

[57] M. Ambrožič, Monte Carlo simulation of Weibull distribution of ceramic strength values, in: Society for Experimental Mechanics, volume 11, Society for Experimental Mechanics Inc, 2008. 